\title{
Towards Correlative Cryo Fluorescence-SEM Imaging of Cryo-Planed Frozen- Hydrated Sea Urchin Embryos
}

\author{
Allessandra DiCorato ${ }^{1}$, Bradley Moreno ${ }^{1}$, and Derk Joester ${ }^{1}$ \\ ${ }^{1 .}$ Materials Science and Engineering, Northwestern University, Evanston, IL, USA.
}

Sea urchin embryos have long inspired scientists for their remarkable ability to synthesize singlecrystalline calcite spicules with a curved shape. The uptake of ions from the sea urchin's environment to form these spicules is a complex process that is not yet fully understood.

Correlative cryogenic scanning electron microscopy (cryoSEM) and fluorescence microscopy enables simultaneous structural and functional or elemental analysis, making it an ideal combination of techniques to study the transport of ions in biological organisms. Previous studies have employed this method to visualize shell mineralization in foraminifera [1] as well as calcium/seawater transport in sea urchin embryos [2]. Despite the wealth of information provided by these studies, however, most rely on the traditional freeze-fracture method of sample preparation for cryoSEM. This technique often renders samples difficult to interpret due to the uncontrollable plane of fracture propagation.

To address this issue, we exploit a correlative cryo-imaging technique to map the distribution of calcium within embryos of the purple sea urchin (Strongylocentrotus purpuratus). To facilitate analysis in cryoSEM, we employ the use of CryoTIGM, or cryogenic triple ion gun milling, a novel sample preparation strategy that uses triple argon beams to clear a large flat plane for analysis [3]. This method offers superior surface flatness compared to conventional freeze fracture sample preparation, and is characterized by higher throughput compared to similar techniques such as cryoFIB. Using CryoTIGM, improper freezing within the blastocoel, likely due to its composition, is evident. Small vacuoles within primary mesenchyme cells (PMCs), demonstrating a similar surface texture, are also present (Fig. 1). This could imply similarity in composition with the fluid in the blastocoel as well.

To enable correlative fluorescence microscopy, we have also adapted this ion milling setup to include a custom-built fluorescence microscope containing long working distance objectives which approach the cryo chamber of the ion miller. This microscope offers superior resolution $(\sim 400 \mathrm{~nm})$ compared to a standard stereoscope. By exposing sea urchin embryos to seawater containing calcein, a fluorescent molecule that binds to calcium without crossing intact cell membranes, the uptake of $\mathrm{Ca}^{2+}$ ions can be visualized as the embryo develops. Fluorescence is evident as a disperse cloud throughout the embryo and within the blastocoel, an outline of the mineralizing spicules, and within the PMCs, which deposit the spicules inside a delimited space called the syncytium (Fig. 2).

Results from correlative cryo-fluorescence imaging using the microscope shown in Fig. 2 will be discussed. This information will shed light on the transport of calcium from the surrounding environment to the syncytium. In a more general sense, by establishing this technique as a valid route to investigating a particular biomineralization pathway, we provide a unique imaging platform with which to analyze similar pathways in other organisms. 
References:

[1] G Khalifa et al, Journal of Structural Biology 196 (2016), p. 155.

[2] N Vidavsky et al, Proceedings of the National Academy of the Sciences 111 (2014), p. 39.

[3] IYT Chang and D Joester, Microscopy and Microanalysis 21 (2015), p. 1616.

[4] IYT Chang and D Joester, Journal of Structural Biology 192 (2015), p. 569.

[5] The authors acknowledge funding from the NSF Major Research Instrumentation program (NSF MRI1229693) and the Northwestern University Materials Research Center (DMR-1121262). Research reported in this publication was supported by the National Institute of General Medical Sciences of the National Institutes of Health under Award Number T32GM105538. AD and BM gratefully acknowledge support from the Ryan Fellowship and the Northwestern University International Institute for Nanotechnology. The content is solely the responsibility of the authors and does not necessarily represent the official views of the National Institutes of Health.
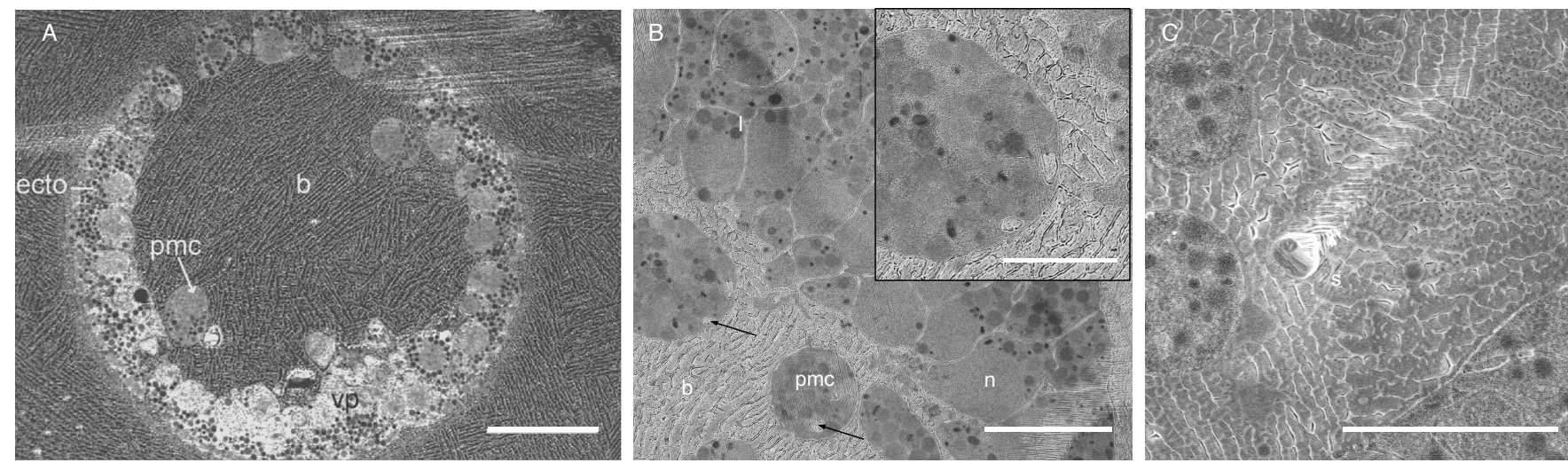

Figure 1. SEM micrographs of frozen-hydrated S. purpuratus sea urchin embryos that have been processed with CryoTIGM. Image in A shows a mesenchyme blastula stage embryo; the blastocoel (b), a primary mesenchyme cell (pmc), and ectoderm (ecto) are visible. Images in B and C show a prism stage embryo. Black arrows indicate possible seawater-filled vesicles inside PMCs, enlarged in inset. The blastocoel (b), spicule (s), primary mesenchyme cells (pmc), lipid-rich vesicles (l) and cell nuclei (n) are visible. Image in A adapted from [4]. Image in B has been processed using the CLAHE plugin in ImageJ. Main scale bars are $20 \mu \mathrm{m}$ in A and $10 \mu \mathrm{m}$ in B and C; inset scale bar is $5 \mu \mathrm{m}$.
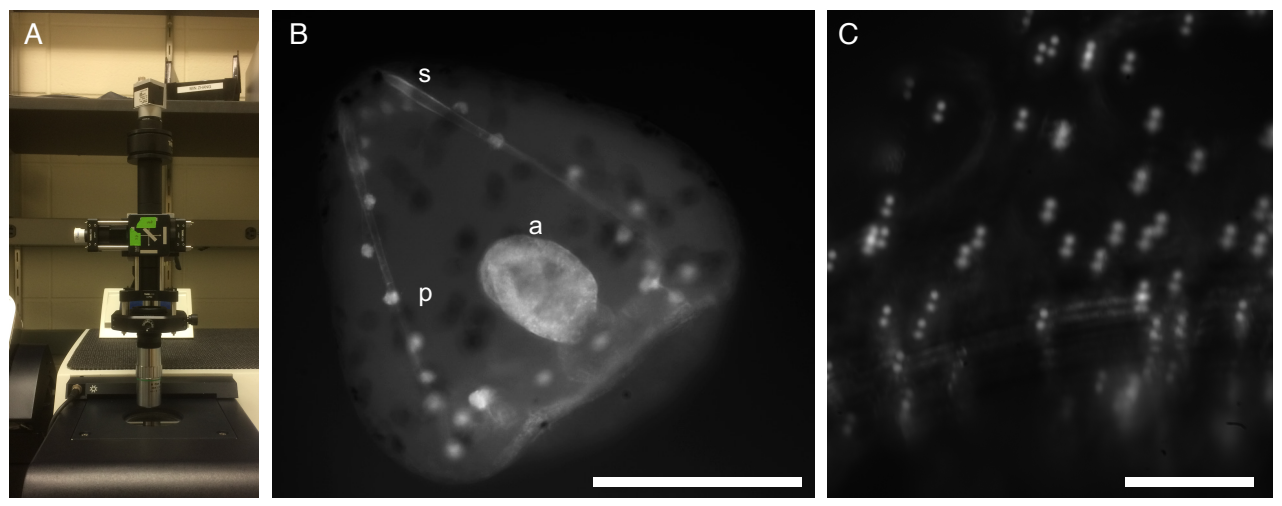

Figure 2. Custom fluorescence microscope (A). Representative room temperature fluorescence image (B) displaying a prism stage $S$. purpuratus sea urchin embryo that has been exposed to calcein-labeled seawater. Evident in the fluorescently-tagged embryo are primary mesenchyme cells (p), the invaginated archenteron (a), and two spicules (s). Image (C) displays a preliminary cryo-fluorescence image depicting an agarose gel containing 6- $\mu \mathrm{m}$ diameter fluorescent microspheres. Scale bars are $100 \mu \mathrm{m}$. 